\title{
Locally delivered GLP-1 analogues liraglutide and exenatide enhance microvascular perfusion in individuals with and without type 2 diabetes
}

\author{
Myo Myo Aung ${ }^{1} \cdot$ Kate Slade $^{1} \cdot$ Leighton A. R. Freeman $^{2} \cdot$ Katarina Kos $^{3} \cdot$ Jacqueline L. Whatmore $^{2}$. \\ Angela C. Shore ${ }^{1,4} \cdot$ Kim M. Gooding ${ }^{1,4}$ (D)
}

Received: 23 January 2019 / Accepted: 24 April 2019 / Published online: 16 June 2019

(C) The Author(s) 2019

\begin{abstract}
Aims/hypothesis Glucagon-like peptide-1 (GLP-1) analogues reduce the risk of macrovascular disease in diabetes; however, little is known about their microvascular effects. This research examined the microvascular actions of the GLP-1 analogues liraglutide and exenatide in individuals with and without type 2 diabetes (study 1 ). It also explored the involvement of the GLP-1 receptor (study 2) and the nitric oxide pathway in mediating the microvascular effects of the analogues.

Methods Trial design: Studies 1 and 2 had a randomised, controlled, double-blind study design. Study 1 participants, intervention and methods: three participant groups were recruited: individuals with well-controlled type 2 diabetes, and obese and lean individuals without diabetes (21 participants per group). Liraglutide $(0.06 \mathrm{mg})$, exenatide $(0.5 \mu \mathrm{g})$ and saline $(154 \mathrm{mmol} / 1 \mathrm{NaCl}$; $0.9 \%$ ) control were microinjected into separate sites in the dermis (forearm) in a randomised order, blinded to operator and participant. Skin microvascular perfusion was assessed by laser Doppler perfusion imaging. Outcomes were stabilised response (mean skin perfusion between 7.5 and $10 \mathrm{~min}$ post microinjection) and total response (AUC, normalised for baseline perfusion). Perfusion response to GLP-1 analogues was compared with saline within each group as well as between groups. Study 2 participants, intervention and methods: in healthy individuals $(N=16)$, liraglutide $(0.06 \mathrm{mg})$ and saline microinjected sites were pretreated with saline or the GLP-1 receptor blocker, exendin- $(9,39)$, in a randomised order, blinded to participant and operator. Outcomes were as above (stabilised response and total perfusion response). Perfusion response to liraglutide was compared between the saline and the exendin- $(9,39)$ pretreated sites. In vitro study: the effects of liraglutide and exenatide on nitrate levels and endothelial nitric oxide synthase phosphorylation (activation) were examined using human microvascular endothelial cells. Results Study 1 results: both analogues increased skin perfusion (stabilised response and total response) in all groups ( $n=21$ per group, $p<0.001)$, with the microvascular responses similar across groups $(p \geq 0.389)$. Study 2 results: liraglutide response (stabilised response and total response) was not influenced by pretreatment with exendin- $(9,39)(70 \mathrm{nmol} / \mathrm{l})(N=15$, one dataset excluded) ( $p \geq 0.609)$. Liraglutide and exenatide increased nitrate production and endothelial nitric oxide synthase (eNOS) phosphorylation $(p \leq 0.020)$.

Conclusions/interpretation Liraglutide and exenatide increased skin microvascular perfusion in individuals with and without well-controlled diabetes, potentially mediated, at least in part, by NO.

Trial registration ClinicalTrials.gov NCT01677104.

Funding This work was supported by Diabetes UK (grant numbers: 09/0003955 and 12/0004600 [RW and JM Collins Legacy, Funded Studentship]).
\end{abstract}

Keywords Exenatide $\cdot$ Glucagon-like peptide-1 analogues $\cdot$ Liraglutide $\cdot$ Microvascular perfusion $\cdot$ Obesity $\cdot$ Type 2 diabetes

Kim M. Gooding

K.M.Gooding@exeter.ac.uk

1 Diabetes and Vascular Medicine, Institute of Biomedical and Clinical Sciences, University of Exeter Medical School, Barrack Road, Exeter EX2 5AX, UK
2 Endothelial Cell Biology Group, University of Exeter Medical School, Exeter, UK

3 Obesity Research Group, University of Exeter Medical School, Exeter, UK

4 National Institute of Health Research Exeter Clinical Research Facility, Royal Devon and Exeter NHS Foundation Trust, Exeter, UK 


\section{Research in context}

\section{What is already known about this subject?}

- Glucagon-like peptide-1 (GLP-1) analogues reduce the risk of macrovascular disease in diabetes

- Previous macrovascular research suggests that GLP-1 analogues may act as vasodilators in humans; however, little is known about their microvascular effects

- Research to date suggests that GLP-1 analogues may increase capillary perfusion/recruitment in individuals without diabetes but not in people with diabetes

\section{What is the key question?}

- Does the local application of the GLP-1 analogues liraglutide and exenatide increase microvascular perfusion and is this effect preserved in obese individuals and people with type 2 diabetes?

\section{What are the new findings?}

- Local delivery of GLP-1 analogues increases skin microvascular perfusion in all groups and the response does not differ with obesity or type 2 diabetes

- Our in vitro experiments suggest that GLP-1 analogues activate the vasodilatory NO pathway in human microvascular cells

- The vasodilatory actions of the GLP-1 analogues may be independent of the GLP-1 receptor

How might this impact on clinical practice in the foreseeable future?

- These data suggest that treatment with GLP-1 analogues has beneficial actions on the microvasculature; these positive effects do not appear to be lost with diabetes or obesity

$\begin{array}{ll}\text { Abbreviations } & \\ \text { ACh } & \text { Acetylcholine } \\ \text { CVD } & \text { Cardiovascular disease } \\ \text { eNOS } & \text { Endothelial nitric oxide synthase } \\ \text { GLP-1 } & \text { Glucagon-like peptide-1 } \\ \text { GLP-1R } & \text { Glucagon-like peptide-1 receptor } \\ \text { LDPI } & \text { Laser Doppler perfusion imaging } \\ \text { LEADER } & \text { Liraglutide Effect and Action in } \\ & \text { Diabetes: Evaluation of Cardiovascular } \\ & \text { Outcome Results } \\ \text { NIHR } & \text { National Institute of Health Research } \\ \text { NREC-SWE } & \text { National Research Ethics Committee } \\ & \text { South West - Exeter } \\ \text { ROI } & \text { Region of interest } \\ \text { SUSTAIN-6 } & \begin{array}{l}\text { Trial to Evaluate Cardiovascular and } \\ \text { Other Long-term Outcomes with }\end{array} \\ & \text { Semaglutide in Subjects with Type } \\ & \text { 2 Diabetes }\end{array}$

\section{Introduction}

Diabetes is associated with vascular complications, which reduce the quality of life of individuals. Therapies that reduce the progression of these vascular complications are critically needed. Glucagon-like peptide-1 $(7,36)$ amide (hereafter referred to as GLP-1) analogues are licensed therapies for type 2 diabetes, aiding glycaemic control by several mechanisms including stimulating postprandial release of insulin and reducing glucagon secretion [1]. Since there is growing evidence that they have beneficial cardiovascular properties, it is important to fully elucidate the effect of GLP-1 analogues on the cardiovascular system in diabetes. This would aid our understanding of their potential clinical role in protecting the vasculature, particularly since they are not first or second line treatments and may guide clinicians in treatment decisions.

The Liraglutide Effect and Action in Diabetes: Evaluation of Cardiovascular Outcome Results (LEADER) and the Trial to Evaluate Cardiovascular and Other Long-term Outcomes with Semaglutide in Subjects with Type 2 Diabetes (SUSTAIN-6) outcome trials demonstrated that GLP-1 analogues, compared with placebo, increased time to cardiovascular event in individuals with type 2 diabetes [2, 3]. The underlying mechanisms are not fully known, although a potential contributing mechanism is a direct action of GLP-1 analogues on the macrovasculature [4].

The impact of GLP-1 analogues on the microvasculature is even less understood, partly due to the scarcity and lack of concordance of available research. An exploratory objective of the LEADER cardiovascular trial was to examine the impact of liraglutide on incidence of microvascular events; results suggest that liraglutide may have differential 
microvascular effects, reducing the incidence of nephropathy events but with no significant effect on retinopathy [2]; similar observations were seen in SUSTAIN-6 [3].

There are few studies examining the microvascular actions of GLP-1 and its analogues. It has been shown that acute, systemic administration of GLP-1 (physiological and supraphysiological levels) increases muscle and cardiac microvessel recruitment in young, healthy, lean individuals $[5,6]$. Additionally, acute, systemic infusion of the GLP-1 analogue exenatide (therapeutic levels) increases the number of perfused dermal capillaries at rest and during reactive hyperaemia in young, healthy, overweight men [7]. Conversely, neither acute administration of exenatide nor short-term liraglutide (12 weeks) influenced capillary perfusion in individuals with type 2 diabetes (fasting and postprandial states) [8]. Interestingly, changes in vasomotion in the skin (dorsal surface of middle finger) were observed in these acute exenatide studies by Smits and colleagues; however, it is difficult to differentiate the systemic effects of exenatide (e.g. increase in heart rate and BP, and reduction in plasma glucose levels) from the local skin microvascular effects. For example, exenatide-induced perfusion changes in the vasomotion neurogenic domain in the fasting state were correlated with glucose, diastolic BP and heart rate in individuals with diabetes [8].

Systemic administration is a widespread impediment to interpreting GLP-1 analogue-related vascular studies, making it difficult to differentiate any direct vascular actions of the analogues from effects due to systemic endocrine (e.g. increased insulin levels), haemodynamic and glycaemic changes. For example, GLP-1 analogues increase circulating levels of insulin, which is known to influence microvascular function [9]. For instance, Tesauro et al [10] observed that acute administration of GLP-1 enhanced endothelial (in)dependent forearm blood flow responses in hyperinsulinaemic conditions in individuals with the metabolic syndrome but had no effect in the absence of hyperinsulinaemia. These difficulties can be overcome by the local, direct administration of the analogues. The skin microvasculature, associated with the coronary circulation [11] and risk of CHD [12], presents an accessible, relevant microvascular bed for examination.

Our limited understanding of the mechanisms underlying the vascular responses to GLP-1 analogues is complicated by the diverse models used and complexity of the potential incretin-influenced vascular pathways. Previous humanbased research (macrovascular cells and digital reactive hyperaemia) proposed that GLP-1 analogue actions are GLP-1 receptor (GLP-1R) dependent [13, 14], as well as NO dependent [13-18] and/or independent [19]. Other proposed mediators in humans include $\mathrm{K}_{\text {ATP }}$ channels [20] and endothelin-1 pathways [15].

The only available study investigating the microvasculature suggested that an exenatide-induced increase in the number of perfused capillaries in healthy, overweight men $(n=10)$ was NO independent [7]. Thus, further research is needed to fully elucidate the effect of GLP-1 analogues on the microvasculature in diabetes to aid our understanding of their potential clinical role in protecting the microcirculation.

Primary aims of this study were to (1) examine the direct, local effect of the GLP-1 analogues, exenatide and liraglutide, on microvascular function; and (2) assess whether the microvascular effects of exenatide and liraglutide differ in lean and obese individuals and in individuals with type 2 diabetes. A secondary aim was to examine whether the GLP-1R and the NO pathways mediate the microvascular actions of GLP-1 analogues. A further aim was to explore whether the microvascular response to liraglutide is associated with clinical characteristics, which may help to identify which individuals show the greatest microvascular benefit.

\section{Methods}

All studies followed the principles of the Declaration of Helsinki. Written, informed consent was obtained from all participants. Microvascular assessments were performed the morning following an overnight fast in a temperaturecontrolled laboratory with the participant in a relaxed, supine position within the National Institute of Health Research (NIHR) Exeter Clinical Research Facility. Participants were recruited via the Peninsula Research Bank, part of the NIHR Exeter Clinical Research Facility.

\section{Study 1: Examining the microvascular actions of exenatide and liraglutide in health and whether this response is altered by obesity and diabetes}

Participants Three participant groups were recruited $(n=21$ per group): lean individuals (BMI $\leq 25.0 \mathrm{~kg} / \mathrm{m}^{2}$ ); obese individuals (BMI $\geq 30.0 \mathrm{~kg} / \mathrm{m}^{2}$ ); individuals with type 2 diabetes. For the lean and obese groups, exclusion criteria included: diabetes; cardiovascular disease (CVD); Raynaud's disease; current treatment with any antihypertensive or lipid-lowering therapies.

For the type 2 diabetes group, individuals on stable diabetes medication (minimum of 3 months) or diet control only were recruited. Exclusion criteria included: insulin or sulfonylurea treatment; known CVD; proliferative retinopathy; advanced nephropathy (macroalbuminuria); uncontrolled diabetes $\left(\mathrm{HbA}_{1 \mathrm{c}}>8.5 \% / 69 \mathrm{mmol} / \mathrm{mol}\right)$; previous GLP-1 analogue or dipeptidyl peptidase-4 inhibitor treatment. The study was approved by the National Research Ethics Committee South West - Exeter (NREC-SWE) (11/SW/0195) and was registered on ClinicalTrials.gov (NCT01677104). 
Methods The study had a randomised, controlled, doubleblind study design.

\section{Participant characterisation and biochemical assessment} methods Participant characterisation: Body composition assessments included height, weight and waist-to-hip ratio. Waist circumference was measured (end of expiration) midway between the costal margin and iliac crest. Hip circumference was measured at the widest horizontal circumference. All measurements were repeated three times and the mean value taken. BP was taken using a semi-automatic device (Dinamap, Critikon, FL, USA) five times at 1 min intervals; the mean of the last three measurements represented BP. A timed, overnight urine sample was collected by all participants to assess for microalbuminuria (AER $>20 \mu \mathrm{g} / \mathrm{min}$ ). Neuropathy was assessed using monofilaments (Semmes Weinstein $10 \mathrm{~g}$ monofilament, Owen Mumford, Woodstock, UK) over six sites on each foot. A score of $\leq 3$ out of 6 in either foot was classed as significant neuropathy.

Biochemical assessments: Plasma glucose, creatinine, triacylglycerols, total cholesterol and HDL levels, and urinary albumin were determined using Modular Analytics, Roche P800 (Roche Diagnostics, Mannheim, Germany). LDL was calculated using the Friedewald formula. $\mathrm{HbA}_{1 \mathrm{c}}$ was determined with the gold standard ion-exchange method (Tosoh G8 HPLC Analyzer, Tosoh Bioscience, San Francisco, CA, USA). Insulin samples were analysed using the Roche E170 chemiluminescent immunoassay (Roche Diagnostics). HOMA insulin resistance was calculated using fasting blood glucose and plasma insulin (HOMA calculator V 2.2.3, 2004, University of Oxford, Diabetes Trial Unit, Oxford, UK). eGFR was calculated using the Modification of Diet in Renal Disease (MDRD) equation [21].

Microvascular assessment Participants with diabetes abstained from medication on the study morning. Fasting blood samples were taken upon arrival.

Microinjection protocol The GLP-1 analogues (one-tenth of the lowest treatment dose, exenatide [0.5 $\mu \mathrm{g}$; Lilly, Basingstoke, UK] and liraglutide [0.06 mg; Novo Nordisk, Gatwick, UK]), acetylcholine (ACh, $1 \%$ [10 mg/ml]; Miochol-E, Bausch \& Lomb, Kingston-Upon-Thames, UK) (positive endothelial function control) and $0.9 \%$ saline (154 mmol/1 NaCl; Fannin, Dublin, UK) (injection trauma control) were delivered by microinjection on the same visit. Exenatide was diluted (1:5) with saline to obtain $0.5 \mu \mathrm{g}$. The order of test sites was blinded and randomised using a random number generator (http://www.stattrek.com).

A black adhesive collar was attached to the volar aspect of the forearm to delineate the region of interest (ROI) $\left(1.54 \mathrm{~cm}^{2}\right)$ for each microinjection site. Each site was at least $2 \mathrm{~cm}$ apart, avoiding visible veins, skin lesions, freckles and hair. A sterile, disposable insulin syringe (30G, BD Microfine, Becton Dickinson, Dublin, Ireland), bent $90^{\circ}$, was used to deliver $10 \mu \mathrm{l}$ of the test substance into the dermis of the ROI. The skin perfusion response was assessed by laser Doppler perfusion imaging (LDPI) (PIM 3.0, Perimed, Järfälla, Sweden) at baseline (resting perfusion) and then every $30 \mathrm{~s}$ for $10 \mathrm{~min}$ post injection. The skin perfusion response is reported as the stabilised response (mean perfusion 7.510 min post injection) and total response (AUC, normalised for resting perfusion). The stabilised response examines the response to the substance of interest following the resolution of the injection trauma perfusion response. Intraparticipant $\mathrm{CV}$ for the stabilised response was $9.7 \%$ (mean $\pm \mathrm{SD}: 1.40$ $\pm 0.13 V)$ for liraglutide and $8.7 \%(1.00 \pm 0.09 V)$ for exenatide, calculated from one lean individual on three separate occasions. This protocol (doses and assessment time) was informed by preliminary experiments. Blood glucose was regularly monitored throughout the study.

\section{Study 2: Examining the role of the GLP-1R in mediating the microvascular actions of liraglutide}

Participants Sixteen healthy (no diabetes, hypertension or CVD), lean individuals (BMI $\leq 25 \mathrm{~kg} / \mathrm{m}^{2}$ ), $>18$ years, were recruited. The exclusion criteria were the same as for the lean group in study 1 . No participants took part in both study 1 and study 2 . The study was approved by the NREC-SWE (14/SW/ 0093).

Study design The study had a randomised, controlled, doubleblind study design. Participant characterisation, biochemical assessments and study conditions were as for study 1 .

Microinjection protocol To examine the impact of GLP-1R inhibition on microvascular response to liraglutide, the ROI was pretreated with exendin- $(9,39)(70 \mathrm{nmol} / \mathrm{l})$ (Bachem, Bubendorf, Switzerland) using a double microinjection protocol. Exendin-(9,39) $(10 \mu \mathrm{l})$ was initially microinjected (first microinjection), followed $60 \mathrm{~s}$ later by liraglutide (10 $\mu 1,0.06 \mathrm{mg})$ (second microinjection) $\sim 1 \mathrm{~mm}$ from the first microinjection site (referred to as Exendin-(9.39) liraglutide site). Three further sites were treated with (1) saline $(0.9 \%)$ followed by liraglutide (referred to as liraglutide site); $(2)$ exendin- $(9,39)$ followed by saline (referred to as exendin- $(9,39)$ site); and (3) saline followed by saline (microinjection trauma control, referred to as saline site). The order of test sites was blinded and randomised as above.

Microinjection, data acquisition and analysis were performed as described for study 1 . The skin perfusion response of each site was assessed by LDPI at baseline, immediately after the first microinjection and every $30 \mathrm{~s}$ for $10 \mathrm{~min}$ following the second microinjection. 
In vitro study: Examining the role of NO in mediating the microvascular actions of GLP-1 analogues

The role of NO in mediating the microvascular actions of GLP-1 analogues was examined in cultured human microvascular endothelial cells (HCMEC/D3 cell line) [22] by assessing the effects of exenatide $(100 \mathrm{pmol} / \mathrm{l})$ (Isca Biochemicals, Exeter, UK) and liraglutide $(10 \mathrm{nmol} / \mathrm{l})$ (Isca Biochemicals) on endothelial nitric oxide synthase (eNOS) activation (phosphorylation) and nitrate levels.

eNOS phosphorylation Cells were treated with medium only (control), exenatide or liraglutide for $10 \mathrm{~min}(n=8$ per condition) and eNOS phosphorylation was determined using a commercially available ELISA kit (eNOS Phospho-Ser 1176 [catalogue No.: OKAG01931], Aviva Systems Biology, San Diego, CA, USA). Phosphorylated levels were normalised to total eNOS and data are expressed as percentage of control.

Nitrate levels Cells were treated with medium only (control), exenatide or liraglutide for $24 \mathrm{~h}$ ( $n=9$ per condition). Supernatants were then collected and stored for nitrate analysis using a Sievers Nitric Oxide Analyzer (Sievers NOA 280, Analytix, Tyne \& Wear, UK) [23]. Results are expressed as percentage of control (medium only).

\section{Statistical analysis}

Data are presented as mean $\pm \mathrm{SD}$ or as median (25th-75th percentile) if data were not normally distributed. Significance was defined as $p \leq 0.05$. In keeping with Cupples et al [24] and Rothman [25], significance is reported without adjustment for multiple testing.

Study 1: 21 individuals per group were recruited, enabling the study to detect a $1 \mathrm{SD}$ difference between groups and a $0.70 \mathrm{SD}$ within-participant difference at $90 \%$ power. For within-group analysis, ANOVAs for repeated measures or Friedman's test, depending on normality, were initially used. Post hoc testing used either paired $t$ tests or Wilcoxon signed rank tests to determine where the difference(s) were (saline vs exenatide, liraglutide or ACh). For between-groups analysis, one-way ANOVAs or Kruskal-Wallis test were initially performed. Post hoc testing used either a Student's $t$ test or a Mann-Whitney $U$ test.

Study 2: 16 individuals were recruited, enabling the study to detect a $0.8 \mathrm{SD}$ within-participant difference at $90 \%$ power. Paired $t$ test or Wilcoxon signed rank test, depending on normality of data, was used to determine whether the GLP-1R inhibition altered the microvascular response to liraglutide (liraglutide site vs exendin-(9,39) liraglutide site).

To examine whether the microvascular actions of liraglutide are associated with clinical and metabolic characteristics (age, body composition, BP, glycaemic control and lipid profile), data from both study 1 and study 2 were merged. The stabilised response to liraglutide across the merged cohorts was initially examined using Spearman's correlation test. Significant associations from univariate analysis, using the variable with the strongest $r$ for each class of characteristics (e.g. BP or lipid profile variable), were further explored using linear regression, adjusting for potential confounding factors (sex and stabilised response to saline control site).

In vitro study: the Mann-Whitney $U$ test was used to compare the responses to exenatide and liraglutide with the responses to control in the in vitro experiments.

\section{Results}

\section{Study 1}

Sixty-three participants completed the study. In the type 2 diabetes group, diabetes was controlled by diet alone in five (24\%) and by metformin in $16(76 \%)$ participants. Median duration of diabetes was 7 (25th-75th percentile: $3-9$ ) years, and $86 \%$ of participants with diabetes were taking cholesterollowering tablets and $57 \%$ antihypertensive treatment. None of the participants (all groups) showed any evidence of microalbuminuria, advanced retinopathy or significant neuropathy.

$\mathrm{HbA}_{1 \mathrm{c}}$ and fasting glucose levels were within the normal range for all participants in the obese group. Insulin and HOMA levels were significantly higher in participants with obesity and diabetes than in lean participants (Table 1). BMI in the obese group was also significantly higher than in the diabetes group.

The direct, local effect of GLP-1 analogues, exenatide and liraglutide, on skin perfusion All microinjections, including saline, caused an initial increase in skin perfusion due to the injection trauma. The response to saline decays over time, presumably due to resolution of this trauma response (representative graph in Fig. 1). Exenatide, liraglutide and ACh significantly increased skin perfusion compared with control in all participant groups ( $p$ values $<0.001$ ). The microinjection protocol was well tolerated by all participants. Blood glucose remained in the normal range throughout the microinjection protocol.

The effects of obesity and diabetes on the microvascular effects of exenatide and liraglutide The microvascular responses to exenatide, liraglutide and ACh (stabilised response and total response) were comparable between the three groups of participants (stabilised and total response to exenatide and liraglutide: $p$ values $\geq 0.456$ and $\geq 0.389$, respectively; stabilised and total response to ACh: $p=0.332$ and 0.250 , respectively) (Fig. 2). 
Table 1 Clinical characteristics of the lean, obese and type 2 diabetes groups in study 1

\begin{tabular}{|c|c|c|c|c|}
\hline Characteristic & Lean group & Obese group & Type 2 diabetes group & $p$ value $^{\mathrm{a}}$ \\
\hline Sample size (\% male) & $21(57)$ & $21(29)$ & $21(43)$ & \\
\hline Age (years) & $65(54-70)$ & $67(52-70)$ & $70(64-71)$ & 0.064 \\
\hline BMI $\left(\mathrm{kg} / \mathrm{m}^{2}\right)$ & $23.00(22.00-24.00)$ & $33.00(31.50-38.00)^{* * *}$ & $30.00(26.25-33.00)^{* * *, \dagger \dagger}$ & $<0.001$ \\
\hline Waist circumference $(\mathrm{cm})$ & $81.69 \pm 6.64$ & $104.93 \pm 10.55^{* * *}$ & $99.73 \pm 9.99 * * *$ & $<0.001$ \\
\hline Waist/hip ratio & $0.83(0.80-0.88)$ & $0.86(0.83-0.97)^{*}$ & $0.91(0.86-0.99)^{* * *}$ & $<0.001$ \\
\hline Systolic BP (mmHg) & $127(118-138)$ & $140(134-154)^{*}$ & $141(134-155)^{*}$ & 0.037 \\
\hline Diastolic BP (mmHg) & $76(70-87)$ & $81(75-88)$ & $77(71-86)$ & 0.221 \\
\hline Mean arterial pressure $(\mathrm{mmHg})$ & $92 \pm 10$ & $99 \pm 10$ & $99 \pm 12$ & 0.082 \\
\hline $\mathrm{HbA}_{1 \mathrm{c}}(\mathrm{mmol} / \mathrm{mol})$ & $39.00 \pm 2.02$ & $38.8 \pm 3.6$ & $48.9 \pm 6.9 * * *, \dagger \dagger$ & $<0.001$ \\
\hline $\mathrm{HbA}_{1 \mathrm{c}}(\%)$ & $5.7 \pm 2.3$ & $5.7 \pm 2.5$ & $6.6 \pm 2.8^{* * *, \dagger \dagger}$ & \\
\hline Fasting blood glucose (mmol/1) & $4.80(4.60-5.33)$ & $5.30(5.05-5.50)^{*}$ & $7.03(6.01-7.74)^{* * *, \dagger \dagger}$ & $<0.001$ \\
\hline Fasting plasma insulin (pmol/l) & $29.33(22.59-37.19)$ & $64.20(54.64-176.80)^{* * *}$ & $84.50(49.30-122.00)^{* * *}$ & $<0.001$ \\
\hline HOMA (insulin resistance) & $0.60(0.40-0.75)$ & $1.20(1.00-2.05)^{* * *}$ & $1.60(1.00-2.30)^{* * *}$ & $<0.001$ \\
\hline Fasting total cholesterol $(\mathrm{mmol} / \mathrm{l})$ & $5.68 \pm 1.01$ & $5.38 \pm 0.90$ & $4.15 \pm 0.96 * * *, \dagger \dagger$ & $<0.001$ \\
\hline HDL (mmol/l) & $1.67(1.50-2.11)$ & $1.38(1.10-1.70)^{*}$ & $1.41(1.26-1.74)^{*}$ & 0.016 \\
\hline LDL (mmol/l) & $3.22 \pm 0.86$ & $3.02 \pm 0.99$ & $2.07 \pm 0.89 * *, \dagger \dagger$ & 0.002 \\
\hline Fasting triacylglycerols $(\mathrm{mmol} / \mathrm{l})$ & $0.83(0.66-1.09)$ & $1.30(1.03-1.76)^{* *}$ & $1.14(0.87-1.69)^{*}$ & 0.004 \\
\hline $\mathrm{eGFR}\left(\mathrm{ml} / \mathrm{min} / 1.73 \mathrm{~m}^{2}\right)$ & $84.0(71.0-86.0)$ & $71.0(62.0-82.0)$ & $81.5(68.3-89.0)$ & 0.552 \\
\hline
\end{tabular}

Data presented as mean (SD) or median (25th-75th percentile)

${ }^{\mathrm{a}} p$ value for between-group analysis across all three groups

$* p<0.05, * * p<0.01, * * * p<0.001$ vs lean group

${ }^{\dagger} p<0.01,{ }^{\dagger \dagger} p<0.001$ vs obese group

\section{Study 2}

Fifteen participants completed the study (one excluded owing to technical issues) (Table 2). Liraglutide significantly increased skin perfusion (stabilised response and total response median [25th-75th percentile]: 1.84 [1.72-2.36] $V$ and 708 [630-853] $V \times \mathrm{s}$, respectively) compared with the saline control site $(0.93$ [0.82-1.35] $V$ and 395 [320-506] $V \times s)(p$ values $=0.001)$ and

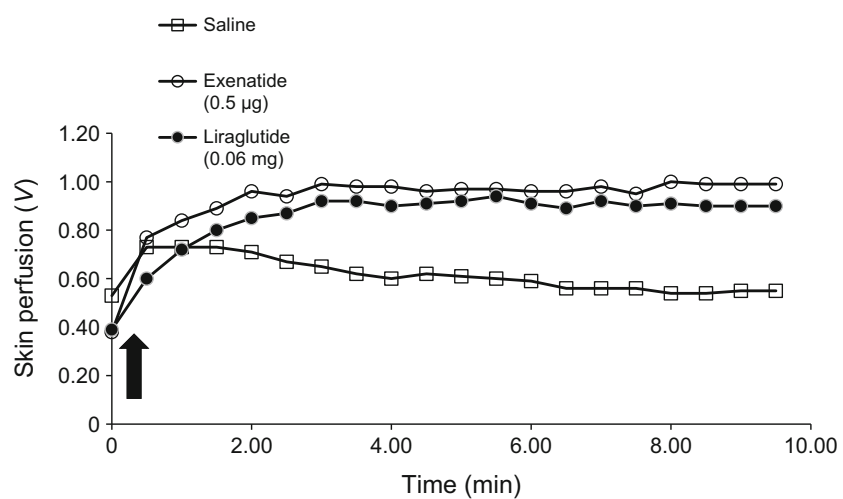

Fig. 1 Representative skin perfusion response to microinjection of the GLP-1 analogues exenatide and liraglutide, compared with saline control, in a lean individual; the graph represents a typical pattern of response that was observed across all participants $(n=63)$. The arrow denotes time of microinjection the response to liraglutide was not altered by pretreatment with exendin-(9,39) (exendin-(9,39) liraglutide site stabilised and total response: 1.82 [1.55-2.27] $V$ and 761 [640-854] $V \times$ s, respectively, $p \geq 0.609$ ) (Fig. 3).

Relationship between the response to liraglutide and clinical and metabolic characteristics As the response to GLP-1 analogues was not altered by diabetes or obesity in study 1 , all of the participants from studies 1 and 2 were collated to examine whether the response to liraglutide was associated with clinical and metabolic characteristics. This resulted in a cohort of 78 participants (44\% male) with an age range of $21-85$ years (Table 3).

The stabilised response to liraglutide was negatively associated with age (Spearman's correlation: $\left.r_{\mathrm{s}}=-0.679, p<0.001\right)$, BP (systolic BP: $r_{\mathrm{s}}=-0.422, p<0.001$; diastolic BP: $r_{\mathrm{s}}=-0.312$, $p=0.005$; mean arterial pressure: $\left.r_{\mathrm{s}}=-0.382, p=0.001\right)$, body composition (waist circumference: $r_{\mathrm{s}}=-0.302, p=$ 0.007; waist/hip ratio: $\left.r_{\mathrm{s}}=-0.251, p=0.026\right)$ and glycaemic control (fasting glucose: $r_{\mathrm{s}}=-0.298, p=0.010 ; \mathrm{HbA}_{1 \mathrm{c}}: r_{\mathrm{s}}=$ $-0.494, p<0.001)$. There was no association between response to liraglutide and the lipid profile.

The observed associations of liraglutide (stabilised response) with age, body composition (waist circumference), $\mathrm{BP}$ (systolic) and glycaemic control $\left(\mathrm{HbA}_{1 \mathrm{c}}\right)$, adjusting for 

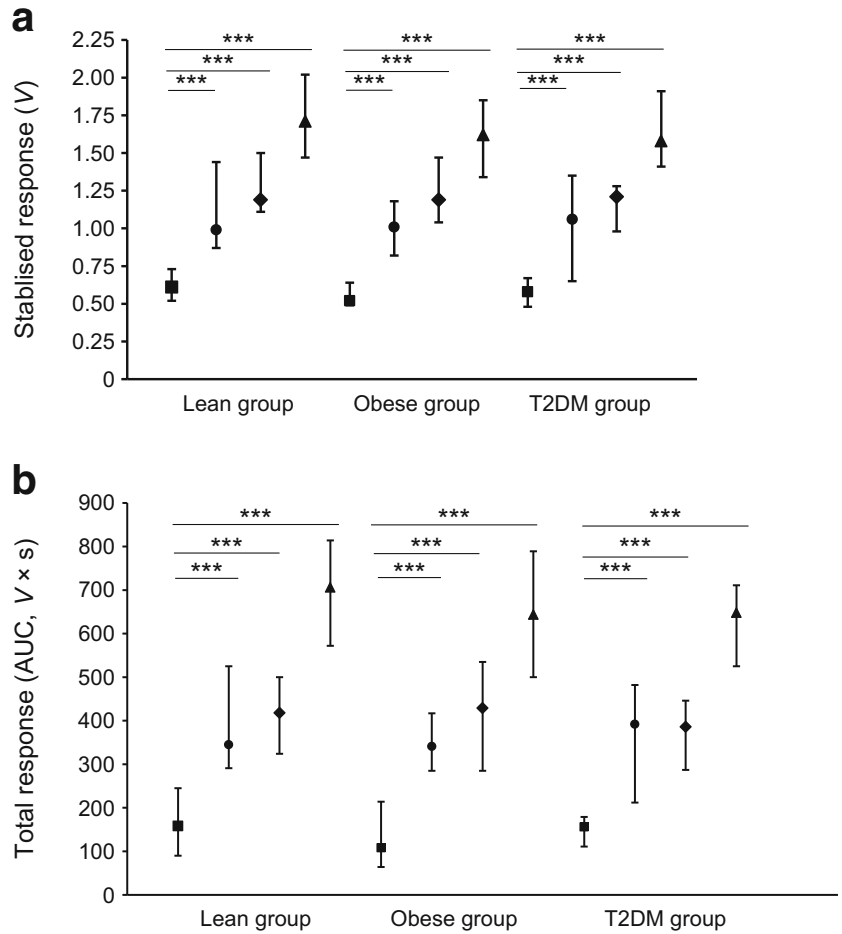

Fig. 2 Skin perfusion response to microinjection of exenatide, liraglutide, ACh and saline in the lean, obese and type 2 diabetes groups $(n=21$ in each group). (a) Stabilised response and (b) total perfusion response to saline (squares), exenatide (circles), liraglutide (diamonds) and ACh (triangles). Data are presented as median (25th-75th percentile). The saline response was significantly lower than the responses to exenatide, liraglutide and $\mathrm{ACh}$, respectively (stabilised response [a] and total response [b]) in all participant groups $(* * * p<0.001$, Wilcoxon signed rank tests). There was no difference in the response to exenatide, liraglutide or ACh between the participant groups. T2DM, type 2 diabetes

Table 2 Characteristics of participants in study 2

\begin{tabular}{ll}
\hline Variable & Value \\
\hline Sample size (\% male) & $16(53)$ \\
Age (years) & $32(22-36)$ \\
BMI $\left(\mathrm{kg} / \mathrm{m}^{2}\right)$ & $22.1 \pm 1.2$ \\
Waist circumference (cm) & $80.7 \pm 7.0$ \\
Waist/hip ratio & $0.82 \pm 0.05$ \\
Systolic BP (mmHg) & $115 \pm 10$ \\
Diastolic BP (mmHg) & $66 \pm 6$ \\
Mean arterial pressure (mmHg) $_{\text {HbA }}(\mathrm{mmol} / \mathrm{mol})$ & $82 \pm 7$ \\
HbA $_{1 \mathrm{c}}(\%)$ & $33.6 \pm 3.0$ \\
Fasting blood glucose (mmol/1) & $5.2 \pm 0.3$ \\
Fasting total cholesterol (mmol/l) & $4.93 \pm 0.31$ \\
HDL (mmol/l) & $4.05 \pm 0.51$ \\
LDL (mmol/l) & $1.60 \pm 0.35$ \\
Fasting triacylglycerols (mmol/l) & $2.05 \pm 0.62$ \\
\hline
\end{tabular}

Data are presented as mean $\pm \mathrm{SD}$ or median (25th-75th percentile)
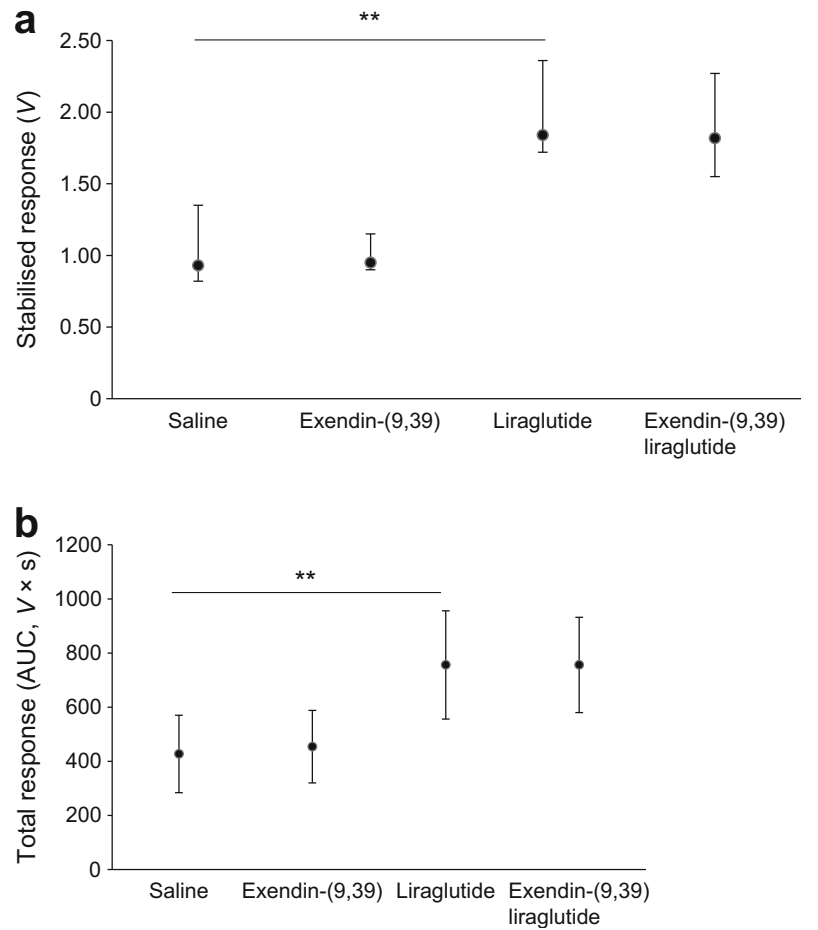

Fig. 3 GLP-1R inhibition does not alter the skin microvascular response to liraglutide in healthy individuals. Each participant $(n=15)$ had 4 treatment sites, each receiving two microinjections: saline site (saline followed by saline); exendin- $(9,39)$ (exendin- $(9,39)$ followed by saline); liraglutide (saline followed by liraglutide); exendin- $(9,39)$ liraglutide (exendin- $(9$, 39) followed by liraglutide). (a) Stabilised response, data presented as median (25th-75th percentile). (b) Total response, data presented as mean (SD). The skin perfusion response at the saline site was significantly lower than the response (stabilised and total) at the liraglutide site (** $p<0.01$ by Wilcoxon signed rank test for stabilised response; by paired $t$ test for total response). Pretreatment by microinjection of exendin- $(9,39)$ did not alter the microvascular response to liraglutide (liraglutide site vs exendin-9,39 liraglutide site, $p \geq 0.609$ by Wilcoxon signed rank test for stabilised response; by paired $t$ test for total response)

sex and response to saline control, were separately explored using linear regression (Table 4). Both age and $\mathrm{HbA}_{1 \mathrm{c}}$, after adjustment for saline response and sex, were individually associated with the response to liraglutide (Table 4). When age, $\mathrm{HbA}_{1 \mathrm{c}}$, sex and response to saline control were included in the model (combined model) they accounted for $50.3 \%$ of the variance of the response to liraglutide (adjusted $R^{2}=0.503$, $F=20.496, p<0.001$ ). Age but not $\mathrm{HbA}_{1 \mathrm{c}}$ was independently associated with the stabilised response to liraglutide in this combined model (Table 4).

\section{In vitro study}

Liraglutide and exenatide significantly increased eNOS phosphorylation compared with control (liraglutide median [25th75th percentile]: 116 [103-119]\%, $p=0.001$; exenatide: 106 [101-110]\%, $p=0.020$; control: $100 \%)$ and nitrate levels compared with control (liraglutide median [25th-75th 
Table 3 Characteristics of participants merged from both study 1 and study 2

\begin{tabular}{ll}
\hline Variable & Value \\
\hline Sample size $(\%$ male) & $78(44 \%)$ \\
Age (years) & $64(46,70)$ \\
BMI $\left(\mathrm{kg} / \mathrm{m}^{2}\right)$ & $24.9(23.0-32.0)$ \\
Waist circumference $(\mathrm{cm})$ & $90.3(81-103)$ \\
Waist/hip ratio & $0.86(0.81-0.92)$ \\
Systolic BP $(\mathrm{mmHg})$ & $135(124-147)$ \\
Diastolic BP $(\mathrm{mmHg})$ & $77 \pm 9$ \\
Mean arterial pressure $(\mathrm{mmHg})$ & $97 \pm 11$ \\
HbA $1 \mathrm{c}(\mathrm{mmol} / \mathrm{mol})$ & $39(37-44)$ \\
HbA1c $(\%)$ & $5.7(5.5-6.2)$ \\
Fasting blood glucose $(\mathrm{mmol} / \mathrm{l})$ & $5.3(4.8-6.0)$ \\
Fasting total cholesterol $(\mathrm{mmol} / \mathrm{l})$ & $4.91 \pm 1.13$ \\
HDL (mmol/l) & $1.56(1.33-1.91)$ \\
LDL (mmol/l) & $2.66 \pm 0.99$ \\
Fasting triacylglycerols $(\mathrm{mmol} / \mathrm{l})$ & $1.06(0.75-1.47)$ \\
\hline
\end{tabular}

Data are presented as mean $\pm \mathrm{SD}$ or median (25th-75th percentile)

percentile]: 201 [178-302]\%, $p=0.002$; exenatide: 169 [140$272] \%, p=0.002$; control: $100 \%$ ) (Fig. 4).

\section{Discussion}

Here, we provide the first evidence of a direct vasodilatory action of GLP-1 analogues in the microcirculation in lean individuals in vivo. Importantly, we also demonstrate that this response is not attenuated in obesity and type 2 diabetes, conditions associated with microvascular impairments $[26,27]$. Furthermore, this research suggests that this increase in microvascular perfusion may be mediated, at least partially, by NO but not via the GLP-1R.

These data suggest that the GLP-1 analogues have a direct vasodilatory effect on the microvasculature (arterioles, venules and capillaries). In contrast, the only previously reported research in this area examined the systemic effects of exenatide or liraglutide on microvascular perfusion in humans, in vivo [7, 8]; an increase in the number of perfused capillaries at rest and during post-occlusive reactive hyperaemia was observed in response to exenatide (continuous i.v. infusion resulting in therapeutic levels) in young (20 27 years), overweight, male participants [7]. Whether this increase in capillary perfusion resulted from a direct action of exenatide is unclear, but results from our study would suggest that this is the case.

We also showed that the microvascular actions of exenatide and liraglutide are maintained in obesity and type 2 diabetes. In contrast, Smits et al [8] observed no changes in capillary perfusion with either acute or short-term treatment with GLP-1 analogues in individuals with relatively well-controlled diabetes $\left(\mathrm{HbA}_{1 \mathrm{c}}\right.$ mean \pm SEM: $7.3 \pm 0.3 \%$ [mean: $56.3 \mathrm{mmol} / \mathrm{mol}$ ]; age: $62.8 \pm 6.9$ years). The participants with type 2 diabetes in the current study (study 1) were slightly older (mean [25th-75th percentile]: 70 [6471] years) with marginally better glycaemic control (6.6 \pm $2.8 \%[48.9 \pm 6.9 \mathrm{mmol} / \mathrm{mol}])$ than those recruited by Smits et al [8]. Since $\mathrm{HbA}_{1 \mathrm{c}}$ was not related to the microvascular responsiveness to GLP-1 analogues and participants were slightly older in our study, when age was negatively related to response, it is unlikely that these variations in participant characteristics could explain the discrepancies between the current study and that of Smits et al [8].

Potential reasons for the observed differences between the diabetes groups in our study and the study by Smits et al [8] may be the delivery route (local vs systemic) and dose (local, dermal delivery at one-tenth minimal treatment dose vs systemic, i.v. administration of therapeutic levels). As detailed earlier, systemic application of GLP-1 analogues at therapeutic doses may result in systemic endocrine, haemodynamic and glycaemic changes, potentially influencing microvascular function including vasomotion [7, 8]. In study 1 , there was a small drop in blood glucose and insulins levels across the study visit (glucose: $\sim 2 \%$; insulin: $18-33 \%$ ), reflecting the participants' fasting state. Importantly, glucose remained in the normal glycaemic range for all participants. Additionally, the site and imaging techniques differed between the two studies (i.e. direct visualisation of digital dermal capillaries vs LDPI of forearm skin microcirculation).
Table 4 Linear regression analysis of stabilised response to liraglutide and clinical and metabolic characteristics

\begin{tabular}{|c|c|c|c|}
\hline Variable & Unstandardised $\beta$ coefficient (SE) & Standardised $\beta$ coefficient & $p$ value \\
\hline \multicolumn{4}{|c|}{ Univariate analysis adjusting for sex and response to saline control } \\
\hline Age & $-0.016(0.003)$ & -0.568 & $<0.001$ \\
\hline Waist & $-0.004(0.003)$ & -0.125 & 0.230 \\
\hline SBP & $-0.003(0.002)$ & -0.144 & 0.178 \\
\hline $\mathrm{HbA}_{1 \mathrm{c}}$ & $-0.019(0.006)$ & -0.285 & 0.003 \\
\hline \multicolumn{4}{|c|}{ Combined model (age, $\mathrm{HbA}_{1 \mathrm{c}}$, sex and response to saline control) } \\
\hline Age & $-0.015(0.003)$ & -0.542 & $<0.001$ \\
\hline $\mathrm{HbA}_{1 \mathrm{c}}$ & $-0.003(0.007)$ & -0.040 & 0.687 \\
\hline
\end{tabular}



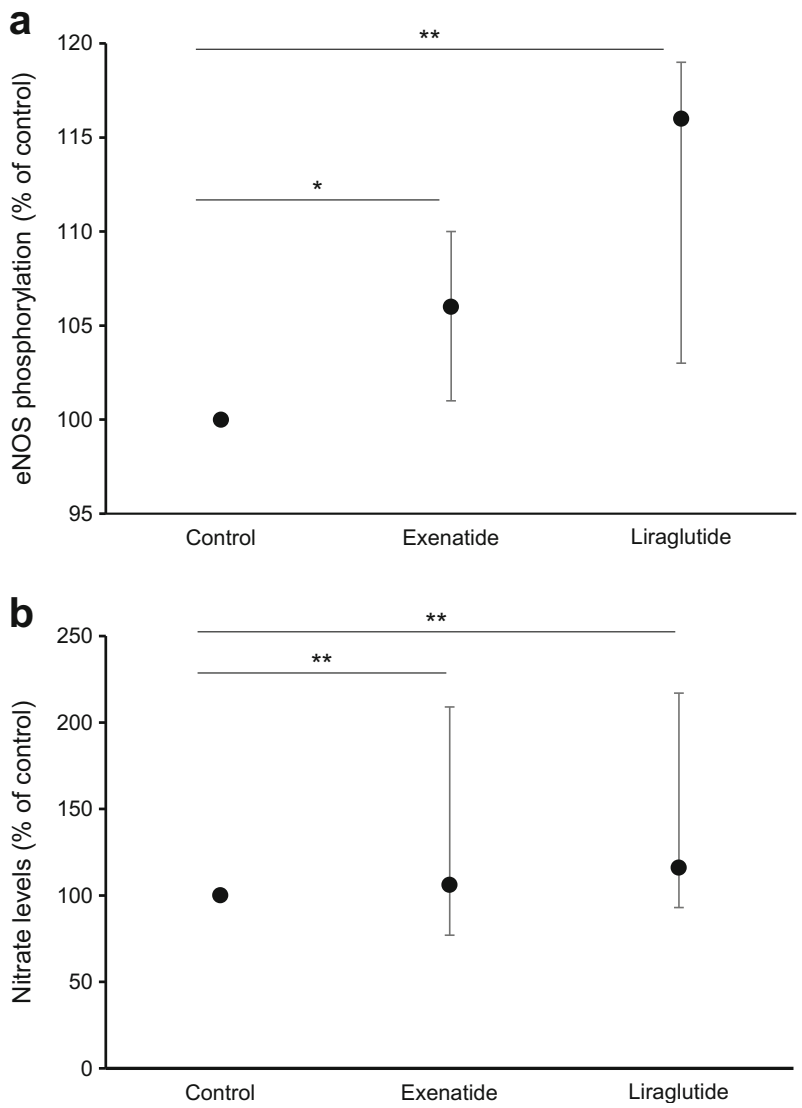

Fig. 4 Exenatide and liraglutide increase eNOS phosphorylation and nitrate levels. (a) eNOS phosphorylation: after initial starvation, human microvascular endothelial cells (HCMEC/D3s) were treated with exenatide and liraglutide for $10 \mathrm{~min}$. Controls were treated with $0.1 \%$ BSA medium only. Phosphorylation data were normalised to total eNOS $(n=8)$. (b) Nitrate levels: HCMEC/D3s were treated with exenatide and liraglutide for $24 \mathrm{~h}(n=9)$. Controls were treated with medium only. For both (a) and (b), data are expressed as percentage of control, with control set at $100 \%$, and are presented as median (25th-75th percentile). ${ }^{*} p<0.05, * * p<0.01$ vs control, Wilcoxon sign rank test.

The strength of the current study is that GLP-1 analogues were delivered locally, ensuring direct microvascular actions without the complication of systemic effects. However, it is important to note that the participants with diabetes had well-controlled glycaemia, no evidence of clinical microvascular complications and good endothelial function, as assessed by ACh microinjection. Whether this conserved response to exenatide and liraglutide in obesity and diabetes reflects that this pathway is intact in these populations, or whether this is partially explained by the relative healthiness of our participants, is unclear. However, the results of the LEADER cardiovascular study [2], which observed vascular benefits in individuals with type 2 diabetes and established, or risk factors for, CVD, may imply that this vascular pathway is intact, although further research is needed to confirm this.
Age was negatively associated with the response to liraglutide. Microvascular function declines with age [28]; however, whether the observed association is due to age-related impairment of microvascular function or specifically due to the GLP-1 pathway is unclear. Since liraglutide is an effective glycaemic treatment in the elderly (>65 years) [29], the observed relationship may relate more to a natural decline in microvascular function with age. This is supported by the association between age and response to $\mathrm{ACh}$ (unstandardised $\beta$ [SE]: -0.011 [0.005]; standardised $\beta$ : $-0.294, p=0.003)$ in this study. Collectively, these data highlight the need to take age into account in human, in vivo studies.

GLP-1Rs have been described in human microvascular endothelial cells [30, 31]. However, the current study suggests that the local microvascular actions of the GLP-1 analogues are GLP-1R independent in healthy humans. This is in contrast to observations by Koska et al [13], who observed an impaired exenatide-induced increase in digital reactive hyperaemia with systemic blockade of the GLP-1R with exendin-(9,39) in participants with impaired glucose tolerance or diet-controlled diabetes. However, the $70 \mathrm{nmol} / \mathrm{l}$ concentration used in study 2 was similar to plasma levels of exendin- $(9,39)$ previously shown to abolish GLP-1-induced insulin secretion and reduce plasma glucose levels in the hyperglycaemic state in healthy participants (plasma mean [SE]: 53 [4] nmol/l) [32].

The NO pathway was upregulated (activation/phosphorylation of eNOS and increased production of nitrate) in human microvascular endothelial cells by both exenatide and liraglutide, suggesting NO involvement in their microvascular actions. This is in agreement with previous studies on the human macrovasculature $[14,16]$, e.g. Koska et al observed a comparable increase in eNOS phosphorylation in human coronary artery endothelial cells [13], but this is the first demonstration in human microvascular endothelial cells. Interestingly, Smits et al observed that exenatide-induced increases in capillary perfusion in healthy, overweight individuals [7] were not modulated by NOS blockade. Since both studies used GLP-1 analogues within their therapeutic ranges, this may reflect differences between in vivo and in vitro studies and/or the heterogeneity of endothelial cells [33]. It is unlikely that the acute nature of the intervention influenced the observations by Smits et al [8] as the current study demonstrated that acute treatment (10 $\mathrm{min}$ ) with GLP-1 analogues increased eNOS activation. The NO pathway may be impaired with obesity [26], raising the possibility that this pathway was downregulated and thus unresponsive in the overweight group recruited by Smits et al [7]. However, the observation that microvascular endothelial function was not impaired in the obese group in the current study suggests that this is not the case. Further research is needed to clarify the involvement of the GLP-1R and the NO pathway in 
mediating the microvascular actions of GLP-1 analogues, particularly in different populations in vivo.

Study limitations: This study may not be applicable to the general diabetes population as only individuals with uncomplicated, well-controlled diabetes were recruited. Additionally, study 2 could have benefited from the inclusion of a control to demonstrate successful GLP-1R inhibition and/or systemic delivery of exendin- $(9,39)$. This research would also be strengthened by extending the NO investigations to examine the effect of NOS blockade in humans, in vivo.

Collectively, our data show that local administration of liraglutide and exenatide increases skin perfusion in lean and obese individuals as well as those with well-controlled, complication-free, type 2 diabetes. It seems likely that this increase in skin perfusion is mediated, at least partially, by an NO-dependent mechanism and independently of the GLP-1R. However, further research is needed to determine whether such local microvascular benefit is preserved in individuals with type 2 diabetes and established microvascular complications or with a systemic increase in GLP-1 levels, such as with clinical treatment with GLP-1 analogues.

Data availability The datasets generated during and/or analysed during the current study are available from the corresponding author on reasonable request.

Funding This work was supported by Diabetes UK (grant numbers: 09/ 0003955 and 12/0004600 [RW and JM Collins Legacy, Funded Studentship]). It was also supported by the National Institute for Health Research (NIHR) Exeter Clinical Research Facility. The views expressed are those of the author(s) and not necessarily those of the NHS, the NIHR or the Department of Health.

Duality of interest The authors declare that there is no duality of interest associated with this manuscript.

Contribution statement MMA contributed to the in vivo study designs, researched data and contributed to the manuscript preparation and discussion. KS contributed to the in vivo (study 2) study design, researched data and contributed to the manuscript discussion. LARF contributed to the in vitro experimental designs, performed in vitro experiments and contributed to the manuscript discussion. KK and ACS contributed to the in vivo study designs, manuscript preparation and discussion. JLW contributed to the in vitro experimental designs, manuscript preparation and discussion. KMG contributed to design of in vivo and in vitro experiments, data analysis and wrote the manuscript. All authors approved the final version of the manuscript. KMG is responsible for the integrity of the data.

Open Access This article is distributed under the terms of the Creative Commons Attribution 4.0 International License (http:// creativecommons.org/licenses/by/4.0/), which permits unrestricted use, distribution, and reproduction in any medium, provided you give appropriate credit to the original author(s) and the source, provide a link to the Creative Commons license, and indicate if changes were made.

\section{References}

1. Kieffer TJ, Habener JF (1999) The glucagon-like peptides. Endocr Rev 20(6):876-913. https://doi.org/10.1210/edrv.20.6.0385

2. Marso SP, Daniels GH, Brown-Frandsen K et al (2016) Liraglutide and cardiovascular outcomes in type 2 diabetes. N Engl J Med 375(4):311-322. https://doi.org/10.1056/NEJMoa1603827

3. Marso SP, Bain SC, Consoli A et al (2016) Semaglutide and cardiovascular outcomes in patients with type 2 diabetes. $\mathrm{N}$ Engl $\mathrm{J}$ Med 375(19):1834-1844. https://doi.org/10.1056/ NEJMoa1607141

4. Selley E, Kun S, Szijarto IA et al (2014) Exenatide induces aortic vasodilation increasing hydrogen sulphide, carbon monoxide and nitric oxide production. Cardiovasc Diabetol 13(1):69. https://doi. org/10.1186/1475-2840-13-69

5. Sjoberg KA, Holst JJ, Rattigan S et al (2014) GLP-1 increases microvascular recruitment but not glucose uptake in human and rat skeletal muscle. Am J Physiol Endocrinol Metab 306(4): E355-E362. https://doi.org/10.1152/ajpendo.00283.2013

6. Subaran SC, Sauder MA, Chai W et al (2014) GLP-1 at physiological concentrations recruits skeletal and cardiac muscle microvasculature in healthy humans. Clin Sci (Lond) 127(3):163-170. https://doi.org/10.1042/CS20130708

7. Smits MM, Muskiet MHA, Tonneijck L et al (2015) GLP-1 receptor agonist exenatide increases capillary perfusion independent of nitric oxide in healthy overweight men. Arterioscler Thromb Vasc Biol 35(6):1538-1543. https://doi.org/10.1161/ATVBAHA.115. 305447

8. Smits MM, Tonneijck L, Muskiet MH et al (2016) GLP-1-based therapies have no microvascular effects in type 2 diabetes mellitus: an acute and 12-week randomized, double-blind, placebocontrolled trial. Arterioscler Thromb Vasc Biol 36(10):21252132. https://doi.org/10.1161/ATVBAHA.116.307930

9. Serne EH, IJzerman RG, Gans RO et al (2002) Direct evidence for insulin-induced capillary recruitment in skin of healthy subjects during physiological hyperinsulinemia. Diabetes 51(5):15151522. https://doi.org/10.2337/diabetes.51.5.1515

10. Tesauro M, Schinzari F, Adamo A et al (2013) Effects of GLP-1 on forearm vasodilator function and glucose disposal during hyperinsulinemia in the metabolic syndrome. Diabetes Care 36(3):683-689. https://doi.org/10.2337/dc12-0763

11. Khan F, Patterson D, Belch JJ, Hirata K, Lang CC (2008) Relationship between peripheral and coronary function using laser Doppler imaging and transthoracic echocardiography. Clin Sci 115(9):295-300. https://doi.org/10.1042/CS20070431

12. IJzerman RG, de Jongh RT, Beijk MA et al (2003) Individuals at increased coronary heart disease risk are characterized by an impaired microvascular function in skin. Eur J Clin Investig 33(7): 536-542. https://doi.org/10.1046/j.1365-2362.2003.01179.x

13. Koska J, Sands M, Burciu C et al (2015) Exenatide protects against glucose- and lipid-induced endothelial dysfunction: evidence for direct vasodilation effect of GLP-1 receptor agonists in humans. Diabetes 64(7):2624-2635. https://doi.org/10.2337/db14-0976

14. Wei R, Ma S, Wang C et al (2016) Exenatide exerts direct protective effects on endothelial cells through the AMPK/Akt/eNOS pathway in a GLP-1 receptor-dependent manner. Am J Physiol Endocrinol Metab 310(11):E947-E957. https://doi.org/10.1152/ajpendo. 00400.2015

15. Dai Y, Mehta JL, Chen MM (2013) Glucagon-like peptide-1 receptor agonist liraglutide inhibits endothelin-1 in endothelial cell by repressing nuclear factor-kappa B activation. Cardiovasc Drugs Ther 27(5):371-380. https://doi.org/10.1007/s10557-013-6463-z 
16. Erdogdu O, Nathanson D, Sjoholm A et al (2010) Exendin-4 stimulates proliferation of human coronary artery endothelial cells through eNOS-, PKA- and PI3K/Akt-dependent pathways and requires GLP-1 receptor. Mol Cell Endocrinol 325(1-2):26-35. https://doi.org/10.1016/j.mce.2010.04.022

17. Hattori Y, Jojima T, Tomizawa A et al (2010) A glucagon-like peptide-1 (GLP-1) analogue, liraglutide, upregulates nitric oxide production and exerts anti-inflammatory action in endothelial cells. Diabetologia 53(10):2256-2263. https://doi.org/10.1007/s00125010-1831-8

18. Muskiet MH, Tonneijck L, Smits MM et al (2016) Acute renal haemodynamic effects of glucagon-like peptide-1 receptor agonist exenatide in healthy overweight men. Diabetes Obes Metab 18(2): 178-185. https://doi.org/10.1111/dom.12601

19. Garczorz W, Francuz T, Siemianowicz K et al (2015) Effects of incretin agonists on endothelial nitric oxide synthase expression and nitric oxide synthesis in human coronary artery endothelial cells exposed to TNF $\alpha$ and glycated albumin. Pharmacol Rep 67(1):6977. https://doi.org/10.1016/j.pharep.2014.08.007

20. Ha SJ, Kim W, Woo JS et al (2012) Preventive effects of exenatide on endothelial dysfunction induced by ischemia-reperfusion injury via KATP channels. Arterioscler Thromb Vasc Biol 32(2):474- 480. https://doi.org/10.1161/ATVBAHA.110.222653

21. Levey AS, Coresh J, Greene T et al (2006) Chronic Kidney Disease Epidemiology Collaboration. Using standardized serum creatinine values in the modification of diet in renal disease study equation for estimating glomerular filtration rate. Ann Intern Med 145(4):247254. https://doi.org/10.7326/0003-4819-145-4-200608150-00004

22. Weksler BB, Subileau EA, Perriere N et al (2005) Blood-brain barrier-specific properties of a human adult brain endothelial cell line. FASEB J 19(13):1872-1874. https://doi.org/10.1096/fj.043458fje

23. Gilchrist M, Winyard PG, Aizawa K, Anning C, Shore A, Benjamin N (2013) Effect of dietary nitrate on blood pressure, endothelial function, and insulin sensitivity in type 2 diabetes. Free Radic Biol Med 60:89-97. https://doi.org/10.1016/j. freeradbiomed.2013.01.024

24. Cupples LA, Heeren T, Schatzkin A, Colton T (1984) Multiple testing of hypotheses in comparing two groups. Ann Intern Med 100(1):122-129. https://doi.org/10.7326/0003-4819-100-1-122

25. Rothman KJ (1990) No adjustments are needed for multiple comparisons. Epidemiology 1(1):43-46. https://doi.org/10.1097/ 00001648-199001000-00010
26. de Jongh RT, Serné EH, IJzerman RG, de Vries G, Stehouwer CD (2004) Impaired microvascular function in obesity: implications for obesity-associated microangiopathy, hypertension, and insulin resistance. Circulation. 109(21):2529-2535. https://doi.org/10.1161/ 01.CIR.0000129772.26647.6F

27. Morris SJ, Shore AC, Tooke JE (1995) Responses of the skin microcirculation to acetylcholine and sodium nitroprusside in patients with NIDDM. Diabetologia 38(11):1337-1344. https://doi.org/10. 1007/BF00401767

28. Minson CT, Holowatz LA, Wong BJ, Kenney WL, Wilkins BW (2002) Decreased nitric oxide- and axon reflex-mediated cutaneous vasodilation with age during local heating. J Appl Physiol 93(5): 1644-1649. https://doi.org/10.1152/japplphysiol.00229.2002

29. Bode BW, Brett J, Falahati A, Pratley RE (2011) Comparison of the efficacy and tolerability profile of liraglutide, a once-daily human GLP-1 analog, in patients with type 2 diabetes $\geq 65$ and $<65$ years of age: a pooled analysis from phase III studies. Am J Geriatr Pharmacother 9(6):423-433. https://doi.org/10.1016/j.amjopharm. 2011.09.007

30. Egholm C, Khammy MM, Dalsgaard T et al (2016) GLP-1 inhibits VEGFA-mediated signaling in isolated human endothelial cells and VEGFA-induced dilation of rat mesenteric arteries. Am J Physiol Heart Circ Physiol 311(5):H1214-H1224. https://doi.org/10.1152/ ajpheart.00316.2016

31. Freeman LAR, Gooding KM, Whatmore JL (2015) Proliferation effects of liraglutide on human microvascular endothelial cells. Diabet Med 32(Suppl 1):100

32. Edwards CM, Todd JF, Mahmoudi M et al (1999) Glucagon-like peptide 1 has a physiological role in the control of postprandial glucose in humans: studies with the antagonist exendin 9-39. Diabetes 48(1):86-93. https://doi.org/10.2337/diabetes.48.1.86

33. Aird WC (2007) Phenotypic heterogeneity of the endothelium: I. Structure, function, and mechanisms. Circ Res 100(2):158-173. https://doi.org/10.1161/01.RES.0000255691.76142.4a

Publisher's note Springer Nature remains neutral with regard to jurisdictional claims in published maps and institutional affiliations. 